\title{
Hematologia de tilápias-do-nilo alimentadas com dietas com óleos vegetais e estimuladas pelo frio
}

\author{
Daniel de Magalhães Araujo(1), Antonio Celso Pezzato(2), Margarida Maria Barros ${ }^{(2)}$, \\ Luiz Edivaldo Pezzato(2) e Fernando Kojima Nakagome(2)
}

\begin{abstract}
(1)Instituto Federal de Alagoas, Departamento de Produção, Pesquisa e Inovação, Rua 17 de Agosto, s/nº, Zona Rural, CEP 57120-000 Satuba, AL. E-mail: danielzootecnista@yahoo.com.br (2)Universidade Estadual Paulista, Departamento de Melhoramento e Nutrição Animal, Fazenda Experimental Lageado, Caixa Postal 560, CEP 18610-000 Botucatu, SP. E-mail: cpezzato@fmvz.unesp.br, mbarros@fmvz.unesp.br, epezzato@fmvz.unesp.br, fernandonakagome@gmail.com
\end{abstract}

Resumo - O objetivo deste trabalho foi avaliar os efeitos de dietas com diferentes relações entre ácidos graxos poliinsaturados ômega-6 e ômega-3 nos parâmetros hematológicos e lipídeos plasmáticos de tilápias-do-nilo, antes e após estímulo pelo frio. Foram utilizados 320 alevinos invertidos para macho $( \pm 7,5 \mathrm{~g})$, distribuídos aleatoriamente em 40 tanques $(250 \mathrm{~L})$ e alimentados com oito dietas: basal (sem adição de óleo), $6 \%$ de óleo de girassol (OG), $5 \%$ de OG $+1 \%$ de óleo de linhaça (OL), $4 \%$ de OG $+2 \%$ OL, $3 \%$ de OG $+3 \%$ de OL, $2 \%$ de OG $+4 \%$ de OL, $1 \%$ de OG $+5 \%$ de OL e $6 \%$ de OL. Os parâmetros hematológicos e os lipídeos plasmáticos foram determinados ao final de 85 dias de cultivo e após três dias de estímulo pelo frio. Não houve efeito das dietas sobre nenhuma das variáveis analisadas no período anterior ao estímulo. O número de leucócitos foi reduzido em peixes alimentados com a dieta $6 \%$ de OL, após estímulo pelo frio. O estímulo pelo frio provocou um declínio do estado geral de saúde, como leucopenia. Tilápias-do-nilo alimentadas com dietas com $6 \%$ de OL apresentaram menor resistência ao frio.

Termos para indexação: Oreochromis niloticus, lipídeos plasmáticos, nutrição de peixes, relação ômega-6/ ômega-3, saúde de peixes.

\section{Hematology of Nile tilapia fed diets with vegetable oils and stimulated by cold}

\begin{abstract}
The objective of this work was to evaluate the effect of diets with different omega- 6 and omega-3 polyunsaturated fatty acid ratios on the hematological parameters and plasma lipids of Nile tilapia before and after cold stress. A total of 320 tilapia juveniles sex-inverted males $( \pm 7.50 \mathrm{~g})$ were randomly distributed into 40 tanks $(250 \mathrm{~L})$ and feed with eight diets: basal (without oil supplementation), $6 \%$ sunflower oil (SO), 5\% $\mathrm{SO}+1 \%$ linseed oil (LO), $4 \% \mathrm{SO}+2 \% \mathrm{LO}, 3 \% \mathrm{SO}+3 \% \mathrm{LO}, 2 \% \mathrm{SO}+4 \% \mathrm{LO}, 1 \% \mathrm{SO}+5 \% \mathrm{LO}$, and $6 \%$ LO. Hematological parameters and plasma lipids were evaluated 85 days after cultivation and three days after cold stress. Diets had no effect on any of the analyzed variables before cold stress. The number of leukocytes decreased in fish fed the 6\% LO diet, after cold stress. Cold stress worsened fish health, causing leukopoenia. Nile tilapia fed $6 \%$ LO diet presented less resistance to cold.
\end{abstract}

Index terms: Oreochromis niloticus, plasma lipids, fish nutrition, omega-6 and omega-3 fatty acid ratio, fish health.

\section{Introdução}

Os lipídeos são a principal forma de armazenamento de energia pelos animais, além de serem constituintes de membranas celulares e excelentes isolantes térmicos, mecânicos e elétricos, que protegem órgãos e células. Os ácidos graxos essenciais são componentes dos lipídos que, por não serem sintetizados pelo organismo, são imprescindíveis nas dietas, pois estão relacionados à saúde da pele, dos sistemas visual e nervoso e ao bom funcionamento de diversos órgãos e sistemas. Esses ácidos são convertidos em eicosanoides e mediadores lipídicos, que incluem prostaglandinas, leucotrienos, tromboxanas e lipoxinas, cuja ausência nas dietas está associada a síndromes que podem levar à morte (Curi et al., 2001).

As temperaturas médias ideais para a criação da tilápia-do-nilo variam de 26 a $28^{\circ} \mathrm{C}$, com mínimas e máximas entre 23 e $33^{\circ} \mathrm{C}$, respectivamente. No Brasil, as temperaturas médias são altas e, em algumas regiões e meses do ano, a temperatura da água de cultivo é inferior a $18^{\circ} \mathrm{C}$ e pode perdurar por vários dias. As temperaturas abaixo de $18^{\circ} \mathrm{C}$ e acima de $30^{\circ} \mathrm{C}$ são exacerbadamente estressantes e devem ser evitadas (Lim \& Webster, 2006).

Pesq. agropec. bras., Brasília, v.46, n.3, p.294-302, mar. 2011 
O papel dos lipídeos na saúde dos peixes pode estar relacionado à manutenção da imunidade frente aos fatores estressantes. Uma resposta imune adequada envolve a modulação da resposta celular por alterações nas propriedades da membrana, como permeabilidade e fluidez, assim como nas enzimas e nos receptores associados à membrana (Montero et al., 2003). O estado de fluidez é um fator importante para manutenção da seletividade e troca de fluidos celulares. Os ácidos graxos poliinsaturados podem auxiliar na adequação do estado de fluidez em baixas temperaturas, por apresentar ponto de fusão muito baixo, sendo considerados lipídeos "anticongelamento" das membranas (Brett \& Navarra-Müller, 1997). A fluidez da membrana depende do balanço apropriado de ácidos graxos saturados e insaturados, como componentes dos fosfolipídeos da membrana (Bell et al., 1986).

A nutrição lipídica exerce influência determinante no desempenho zootécnico e nas características da carcaça dos animais em geral, inclusive dos peixes. É possível avaliar o estado nutricional dos peixes por meio de análise das características hematológicas, pois o sangue é um dos tecidos mais dinâmicos do organismo e altera-se em função do tipo de dieta consumida. De acordo com Ranzini-Paiva \& Silva-Souza (2004), a aplicação da hematologia em pesquisas com animais é bem aceita e considerada como procedimento de rotina em métodos de diagnóstico. Em peixes, este tipo de diagnóstico vem sendo aperfeiçoado desde a década de 1990, mas novos estudos ainda são necessários (Feldman et al., 2006), por se tratar de uma importante ferramenta para o diagnóstico, não somente de estados patológicos, mas do estado nutricional dos peixes. Barros et al. (2009) afirmam que, além de nutrir o animal para máximo desempenho, é preciso nutrir seu sistema de defesa.

Foram realizados experimentos com peixes em condições patológicas e de estresse, entretanto, poucos estudos correlacionam valores de referência de peixes saudáveis, o que constitui uma lacuna para a utilização da hematologia (Hrubec et al., 2006). As características hematológicas normais de peixes sadios também devem ser conhecidas para que o diagnóstico do seu estado de saúde ou nutricional seja confiável, principalmente em virtude da ampla variação fisiológica de cada espécie, em cada ambiente de criação, que é representada pela variação causada por fatores bióticos e abióticos (Ranzini-Paiva \& Silva-Souza, 2004). O conhecimento dos valores médios dos parâmetros hematológicos em ambiente natural e em cativeiro, nos mais diversos sistemas de criação comercial, sob condições de homeostase e de estresse, é importante para identificar as alterações fisiológicas, derivadas da nutrição e de fatores ambientais, como a temperatura, que possam interferir na hematopoiese.

O objetivo deste trabalho foi avaliar os efeitos de dietas com diferentes relações entre ácidos graxos poliinsaturados ômega- 6 e ômega- 3 nos parâmetros hematológicos e lipídeos plasmáticos de tilápias-do-nilo antes e após estímulo pelo frio.

\section{Material e Métodos}

O estudo foi conduzido na Universidade Estadual Paulista, campus de Botucatu, SP, no Laboratório de Nutrição de Organismos Aquáticos do Departamento de Melhoramento Zootécnico e Nutrição Animal.

Foram utilizados alevinos de tilápia-do-nilo, invertidos sexualmente para machos, com 7,5 $\pm 0,21 \mathrm{~g}$, distribuídos em 40 tanques experimentais com capacidade de $250 \mathrm{~L}$, em densidade de oito alevinos por aquário, o que totalizou 320 peixes.

Os aquários estavam ligados em sistema de circulação de água, com aquecimento controlado por sistema digital integrado, para manter a temperatura da água constante $\left(26^{\circ} \mathrm{C}\right)$. $\mathrm{O}$ sistema de circulação estava acoplado à central de aeração e filtragem físico-biológica, para manutenção da qualidade da água.

Os tratamentos consistiram de oito rações experimentais: basal (sem adição de óleo), $6 \%$ de óleo de girassol (OG), $5 \%$ de $\mathrm{OG}+1 \%$ de óleo de linhaça (OL), $4 \%$ de $\mathrm{OG}+2 \%$ OL, $3 \%$ de $\mathrm{OG}+3 \%$ de OL, $2 \%$ de $\mathrm{OG}+4 \%$ de OL, $1 \%$ de OG $+5 \%$ de OL e $6 \%$ de OL. A composição percentual e químico-bromatológica das dietas experimentais está apresentada na Tabela 1.

Para confecção das rações, os ingredientes foram moídos em partículas menores que $0,07 \mathrm{~mm}$, homogeneizados manualmente antes e após serem umedecidos, em 18 a $20 \%$ do peso, com água aquecida a $55^{\circ} \mathrm{C}$, sendo posteriormente extrusados. Após extrusão, as rações foram secas em estufa com circulação forçada de ar por 24 horas, a $55^{\circ} \mathrm{C}$, e, em seguida, retiradas, resfriadas à temperatura ambiente, identificadas conforme o tratamento, ensacadas e armazenadas em freezer a $-10^{\circ} \mathrm{C}$ até o uso. Para o arraçoamento, as rações foram moídas e peneiradas

Pesq. agropec. bras., Brasília, v.46, n.3, p.294-302, mar. 2011 
até obtenção de grânulos homogêneos adequados ao tamanho da boca dos peixes. O arraçoamento foi realizado quatro vezes ao dia (às $8 \mathrm{~h} 30 \mathrm{~min}, 11 \mathrm{~h} 30 \mathrm{~min}$, $14 \mathrm{~h} 30 \mathrm{~min}$ e $17 \mathrm{~h} 30 \mathrm{~min}$ ), até aparente saciedade e para evitar sobras.

Os aquários foram sifonados e o sistema de contenção de matéria orgânica do filtro físico-biológico foi lavado quando necessário, para retirar o excesso de matéria orgânica do sistema e manter a qualidade da água. A temperatura da água, o oxigênio dissolvido e o $\mathrm{pH}$ foram mantidos a $25,8 \pm 1,1^{\circ} \mathrm{C}, 6,7 \pm 0,9 \mathrm{mg} \mathrm{L}^{-1} \mathrm{e}$ $7,3 \pm 0,6$, respectivamente, de acordo com os padrões estabelecidos como ideais para a espécie (Boyd \& Tucker, 1998). A temperatura, o oxigênio e o pH foram aferidos duas vezes ao dia, a cada semana e a cada 15 dias, respectivamente.

Aos 85 dias de cultivo, seis peixes $(125,34 \pm 9,69 \mathrm{~g})$ por tratamento foram retirados aleatoriamente das cinco

Tabela 1. Composição percentual e nutricional calculada das rações experimentais ${ }^{(1)}$.

\begin{tabular}{|c|c|c|c|c|c|c|c|c|}
\hline Ingredientes & $\mathrm{B}$ & $6 \mathrm{G}$ & 5G:1L & 4G:2L & $3 \mathrm{G}: 3 \mathrm{~L}$ & $2 \mathrm{G}: 4 \mathrm{~L}$ & 1G:5L & $6 \mathrm{~L}$ \\
\hline Farelo de soja (47\% de PB) & 50,00 & 50,00 & 50,00 & 50,00 & 50,00 & 50,00 & 50,00 & 50,00 \\
\hline Glúten de milho ( $60 \%$ de $\mathrm{PB})$ & 6,00 & 6,00 & 6,00 & 6,00 & 6,00 & 6,00 & 6,00 & 6,00 \\
\hline Quirera de arroz & 17,00 & 12,50 & 12,50 & 12,50 & 12,50 & 12,50 & 12,50 & 12,50 \\
\hline Farelo de trigo & 7,00 & 8,00 & 8,00 & 8,00 & 8,00 & 8,00 & 8,00 & 8,00 \\
\hline Amido de milho & 14,06 & 11,54 & 11,54 & 11,54 & 11,54 & 11,54 & 11,54 & 11,54 \\
\hline Óleo de girassol & 0,00 & 6,00 & 5,00 & 4,00 & 3,00 & 2,00 & 1,00 & 0,00 \\
\hline Óleo de linhaça & 0,00 & 0,00 & 1,00 & 2,00 & 3,00 & 4,00 & 5,00 & 6,00 \\
\hline DL-metionina & 0,65 & 0,67 & 0,67 & 0,67 & 0,67 & 0,67 & 0,67 & 0,67 \\
\hline Triptofano & 0,10 & 0,10 & 0,10 & 0,10 & 0,10 & 0,10 & 0,10 & 0,10 \\
\hline Treonina & 0,42 & 0,42 & 0,42 & 0,42 & 0,42 & 0,42 & 0,42 & 0,42 \\
\hline Fosfato bicálcico & 3,60 & 3,60 & 3,60 & 3,60 & 3,60 & 3,60 & 3,60 & 3,60 \\
\hline Calcáreo & 0,20 & 0,20 & 0,20 & 0,20 & 0,20 & 0,20 & 0,20 & 0,20 \\
\hline Vitamina $\mathrm{C}$ & 0,05 & 0,05 & 0,05 & 0,05 & 0,05 & 0,05 & 0,05 & 0,05 \\
\hline Cloreto de colina & 0,40 & 0,40 & 0,40 & 0,40 & 0,40 & 0,40 & 0,40 & 0,40 \\
\hline $\mathrm{NaCl}$ & 0,10 & 0,10 & 0,10 & 0,10 & 0,10 & 0,10 & 0,10 & 0,10 \\
\hline Suplemento vitamínico ${ }^{(2)}$ & 0,10 & 0,10 & 0,10 & 0,10 & 0,10 & 0,10 & 0,10 & 0,10 \\
\hline Suplemento mineral ${ }^{(3)}$ & 0,30 & 0,30 & 0,30 & 0,30 & 0,30 & 0,30 & 0,30 & 0,30 \\
\hline Etoxiquin $^{(4)}$ & 0,02 & 0,02 & 0,02 & 0,02 & 0,02 & 0,02 & 0,02 & 0,02 \\
\hline Total & 100,00 & 100,00 & 100,00 & 100,00 & 100,00 & 100,00 & 100,00 & 100,00 \\
\hline Massa de matéria seca (\%) & 96,75 & 97,95 & 97,87 & 97,44 & 97,26 & 97,22 & 97,04 & 96,75 \\
\hline Proteína bruta (\%) & 32,53 & 32,27 & 32,27 & 32,27 & 32,27 & 32,27 & 32,27 & 32,27 \\
\hline Proteína digestível & 30,08 & 29,93 & 29,93 & 29,93 & 29,93 & 29,93 & 29,93 & 29,93 \\
\hline Energia digestível (kcal kg-1) & 3.420 & 3.420 & 3.420 & 3.420 & 3.420 & 3.420 & 3.420 & 3.420 \\
\hline Energia digestível/proteína digestível & 113,7 & 114,27 & 114,27 & 114,27 & 114,27 & 114,27 & 114,27 & 114,27 \\
\hline Extrato etéreo $(\%)$ & 2,57 & 8,17 & 8,17 & 8,17 & 8,17 & 8,17 & 8,17 & 8,17 \\
\hline Cálcio (\%) & 1,12 & 1,12 & 1,12 & 1,12 & 1,12 & 1,12 & 1,12 & 1,12 \\
\hline Fósforo digestível (\%) & 0,70 & 0,69 & 0,69 & 0,69 & 0,69 & 0,69 & 0,69 & 0,69 \\
\hline Cálcio + fósforo digestível & 1,61 & 1,63 & 1,63 & 1,63 & 1,63 & 1,63 & 1,63 & 1,63 \\
\hline Metionina + cistina digestível $(\%)$ & 0,96 & 0,98 & 0,98 & 0,98 & 0,98 & 0,98 & 0,98 & 0,98 \\
\hline Lisina digestível (\%) & 2,08 & 2,08 & 2,08 & 2,08 & 2,08 & 2,08 & 2,08 & 2,08 \\
\hline Triptofano digestível (\%) & 0,38 & 0,38 & 0,38 & 0,38 & 0,38 & 0,38 & 0,38 & 0,38 \\
\hline Treonina digestível (\%) & 1,28 & 1,28 & 1,28 & 1,28 & 1,28 & 1,28 & 1,28 & 1,28 \\
\hline AAS digestível (\%) & 1,35 & 1,36 & 1,36 & 1,36 & 1,36 & 1,36 & 1,36 & 1,36 \\
\hline Fibra bruta $(\%)$ & 4,36 & 4,41 & 4,41 & 4,41 & 4,41 & 4,41 & 4,41 & 4,41 \\
\hline \multicolumn{9}{|l|}{ Ácidos graxos calculados (\%) } \\
\hline Ômega-6 & 0,53 & 4,47 & 3,94 & 3,41 & 2,88 & 2,35 & 1,82 & 1,29 \\
\hline Ômega-3 & 0,08 & 0,07 & 0,60 & 1,14 & 1,67 & 2,20 & 2,74 & 3,27 \\
\hline Ômega-6/ômega-3 & 6,62 & 63,85 & 6,56 & 3,00 & 1,72 & 1,07 & 0,66 & 0,39 \\
\hline
\end{tabular}

${ }^{(1)} \mathrm{B}$, dieta basal sem adição de óleo; G, óleo de girassol; L, óleo de linhaça; PB, proteína bruta; AAS; ácido acetilsalicílico. ${ }^{(2)}$ Níveis de garantia por quilograma da dieta: 16.060 UI de vitamina A; 4.510 UI de vitamina D3; 250 UI de vitamina E; 30 mg de vitamina K; 32 mg de vitamina B1; 32 mg de vitamina B2; $80 \mathrm{mg}$ de pantotenato de cálcio; $170 \mathrm{mg}$ de niacina; $10 \mathrm{mg}$ de biotina; $10 \mathrm{mg}$ de ácido fólico; $32 \mu \mathrm{g}$ de vitamina B12; $32 \mathrm{mg}$ de vitamina B6. ${ }^{(3)}$ Níveis de garantia por quilograma da dieta: $0,7 \mathrm{mg} \mathrm{de} \mathrm{Na}_{2} \mathrm{SeO}_{3} ; 50 \mathrm{mg} \mathrm{de} \mathrm{MnO} ; 150 \mathrm{mg}$ de $\mathrm{ZnO} ; 150 \mathrm{mg} \mathrm{de} \mathrm{FeSO} 4 ; 20 \mathrm{mg}$ de $\mathrm{CuSO}_{4}$; 0,5 mg de $\mathrm{CoSO}_{4}$; $1 \mathrm{mg}$ de I2Ca. ${ }^{(4)}$ Antioxidante etoxi-dihidro-trimetilquinolina. 
repetições e anestesiados com benzocaína (1,5 $\mathrm{g}$ do anestésico em 15 L de água) e, após insensibilização, foram coletados $2 \mathrm{~mL}$ de sangue de cada peixe, por punção do vaso caudal, com auxílio de duas seringas de $1 \mathrm{~mL}$ banhadas com anticoagulante (EDTA 3\%).

Foi realizado hemograma dos peixes com o sangue coletado em uma das seringas. A contagem do número de eritrócitos e leucócitos foi realizada pelo método do hemocitômetro, em câmara de Neubauer, com azul de toluidina (Merck Química, Brooklin Novo, $\mathrm{SP}$ ), a $0,01 \%$, diluído em solução fisiológica a $0,9 \%$, com pipeta de Thoma, na proporção de 1:200. A taxa de hemoglobina $(\mathrm{Hb})$ foi determinada pelo método da cianometahemoglobina, com o kit comercial Analisa Diagnóstica (Gold Analisa Diagnóstica Ltda, Belo Horizonte, MG), para determinação colorimétrica, segundo Collier (1944). A percentagem de hematócrito $(\mathrm{Htc})$ foi determinada pelo método do microhematócrito, de acordo com Goldenfarb et al. (1971). A proteína plasmática total (PPT) foi mensurada pelo refratômetro manual de Goldberg, com quebra do tubo de microhematócrito logo acima da camada de leucócitos, após leitura do hematócrito (Jain, 1986). Posteriormente, foram calculados os índices hematimétricos, conforme Wintrobe (1934), como descritos a seguir:

Volume corpuscular médio [VCM $=($ Htc x 10)/ eritrócitos]; concentração de hemoglobina corpuscular média $[\mathrm{CHCM}=(\mathrm{Hb} / \mathrm{Htc}) \times 100]$.

O sangue coletado na segunda seringa foi transferido para tubo de Eppendorf previamente identificado e, em seguida, centrifugado a $2.380 \mathrm{~g}$ por $15 \mathrm{~min}$, a $0^{\circ} \mathrm{C}$. Após centrifugação, o plasma foi armazenado em freezer, a $-20^{\circ} \mathrm{C}$, até determinação de lipídeos totais (LT), colesterol total (COLT), colesterol HDL (C-HDL) e triglicerídeos (TRG) séricos. Os LT foram determinados por ensaio colorimétrico, pela ação da sulfofosfovanilina, segundo Frings et al. (1972). O COLT e os TRG foram determinados por ensaio enzimático-colorimétrico em espectrofotômetro semiautomático (Labtest Diagnóstica S.A, Lagoa Santa, MG), do mesmo modo que o C-HDL, após precipitação de partículas de colesterol não HDL, todos com kits comerciais (Katal Biotecnológica Indústria e Comércio Ltda., Belo Horizonte, MG).

Após as avaliações hematológicas realizadas antes do estimulo pelo frio, os peixes utilizados foram descartados e o restante permaneceu na mesma estrutura. O sistema de aquecimento foi desligado até que houvesse o abaixamento gradativo da temperatura da água, para que esta se igualasse à da sala de estímulo pelo frio, o que ocorreu, aproximadamente, em 24 horas. Quando a temperatura da água dos aquários de ambas as estruturas experimentais estava por volta de $22,5^{\circ} \mathrm{C}$, os peixes foram transferidos para a sala de estímulo pelo frio. A temperatura da água dos aquários passou a ser controlada pelo resfriamento do ar, com condicionador de ar, para abaixamento da temperatura da água para $17^{\circ} \mathrm{C}$, o que ocorreu, aproximadamente, 18 horas após a transferência dos peixes.

Para estímulo pelo frio, foram transferidos seis peixes $(125,34 \pm 9,69 \mathrm{~g})$ de cada tratamento, retirados aleatoriamente das cinco repetições, tendo sido utilizado, ao menos, um peixe de cada uma das repetições. Foram alojados dois peixes por aquário, 48 peixes em 24 aquários de $40 \mathrm{~L}$ dotados de filtro e aeração individuais. Cada aquário foi dividido em dois, com uma tela disposta na diagonal, para evitar confrontos entre os peixes. O período de estímulo pelo frio foi de três dias; entretanto, os peixes ficaram expostos a $17^{\circ} \mathrm{C}$ por, aproximadamente, 54 horas. Após este período, foram avaliadas as mesmas variáveis sanguíneas estudadas ao final dos 85 dias de cultivo e foi mantido o delineamento experimental utilizado previamente.

No período de estímulo pelo frio, o arraçoamento dos peixes foi efetuado duas vezes ao dia (às $8 \mathrm{~h}$ e às 17h), até aparente saciedade e para evitar sobras, como no período anterior.

Para efeito do estudo dos parâmetros hematológicos e dos lipídeos sanguíneos, cada peixe foi considerado uma repetição, sendo que cada média apresentada, antes ou após estímulo pelo frio, representa a média de seis repetições, ou seja, de seis peixes, por tratamento. As médias das variáveis estudadas foram submetidas à análise multivariada de variância (MANOVA), em modelo de medidas repetidas em grupos independentes (Johnson \& Wichern, 2002).

\section{Resultados e Discussão}

No período de 85 dias de cultivo, anterior ao estímulo pelo frio, houve morte de sete peixes nos tratamentos 6G (1), 5G:1L (1), 2G:4L (2), 1G:5L (1) e 6L (2). Nos dias sob estímulo pelo frio, não houve mortes. Também não houve nenhum efeito das dietas 
sobre a mortalidade dos peixes. No período anterior ao estímulo pelo frio, não houve efeito das dietas sobre as variáveis hematológicas; entretanto, durante o estímulo pelo frio, as dietas exerceram influência no número de leucócitos dos peixes. Ao comparar-se os períodos (antes e após estímulo pelo frio), observouse que o estímulo pelo frio promoveu a redução dos valores médios da contagem de eritrócitos, da taxa de hemoglobina, da contagem de leucócitos e da concentração de PPT nos peixes (Tabela 2).

Não houve variação de nenhum dos parâmetros hematológicos das tilápias que consumiram dietas com óleo de milho e girassol, como fontes de ômega-6, ou com óleo de linhaça. Yildirim-Aksoy et al. (2007) encontram resultados semelhantes ao deste trabalho, ao estudar os efeitos do consumo de rações com $7 \%$ de óleos de milho, peixe (menhaden), linhaça ou sebo bovino e suas misturas equivalentes nos parâmetros hematológicos e na resposta imune de tilápias-do-nilo. $\mathrm{O}$ número de eritrócitos e leucócitos foi maior nos peixes alimentados com dietas que continham apenas óleo de peixe, mas não diferiram entre nenhum dos outros tratamentos. Quanto à taxa de hemoglobina e percentual de hematócrito, os autores não observaram efeito do tipo de lipídeo consumido pelos peixes.

O número de eritrócitos foi reduzido após estímulo pelo frio apenas nos peixes que consumiram a dieta 4G:2L. Como estas células são constituintes da série vermelha sanguínea e contêm hemoglobina, cuja função é o transporte de $\mathrm{O}_{2}$ e de parte de $\mathrm{CO}_{2}$, qualquer deficiência em número ou forma dos eritrócitos pode comprometer a oxigenação nos tecidos (Ranzini-Paiva \& Silva-Souza, 2004).

Não foi observada redução dos valores de hematócrito em peixes alimentados com dietas com óleo de linhaça, submetidos ou não ao frio. Os demais valores de hematócrito (Tabela 2), para peixes antes e após estímulo pelo frio, com exceção do mínimo após estímulo pelo frio, estão entre os considerados normais (27 a 37\%) para tilápias saudáveis por Feldman et al. (2006).

A taxa de hemoglobina foi reduzida nos peixes que consumiram ração $6 \mathrm{~L}$ após estímulo pelo frio e pode ser alterada em função do número de eritrócitos, o que não ocorreu neste trabalho. A redução da hemoglobina pode significar menor capacidade de transporte de gases no interior do corpo. No geral, quanto menor a temperatura, maior a concentração de oxigênio dissolvido na água. Quando a temperatura é inferior à ótima para peixes tropicais, os peixes reduzem seu metabolismo, principalmente em amplitudes relativamente grandes, como neste trabalho, em que houve diminuição de cerca de $9^{\circ} \mathrm{C}$. A combinação dessas duas situações pode explicar a redução da taxa de hemoglobina circulante no sangue periférico, decorrente da aclimatação dos peixes, por haver menor demanda metabólica por oxigênio e mais oxigênio disponível para respiração em água mais fria. A redução observada foi mínima e os valores absolutos antes e após estímulo pelo frio estão muito próximos

Tabela 2. Parâmetros hematológicos $(n=6)$ de tilápias-do-nilo alimentadas com rações com óleos de girassol, linhaça e suas misturas antes e após estímulo pelo frio.

\begin{tabular}{|c|c|c|c|c|c|c|c|c|c|c|}
\hline \multirow[t]{2}{*}{ Tratamento $^{(1)}$} & \multicolumn{2}{|c|}{ Eritrócito $\left(10^{6} \mu \mathrm{L}^{-1}\right)$} & \multicolumn{2}{|c|}{ Hematócrito (\%) } & \multicolumn{2}{|c|}{ Hemoglobina $\left(\mathrm{g} \mathrm{dL}^{-1}\right)$} & \multicolumn{2}{|c|}{ Leucócitos $\left(10^{5} \mu \mathrm{L}^{-1}\right)$} & \multicolumn{2}{|c|}{$\mathrm{PPT}^{(4)}\left(\mathrm{g} \mathrm{dL}^{-1}\right)$} \\
\hline & Antes & Após & Antes & Após & Antes & Após & Antes & Após & Antes & Após \\
\hline Basal & $1,99 \pm 0,25$ & $1,94 \pm 0,20$ & $30,67 \pm 3,54$ & $29,25 \pm 2,40$ & $7,27 \pm 0,96$ & $7,34 \pm 0,46$ & $1,37 \pm 0,35$ & $1,01 \pm 0,24 \mathrm{~A}$ & $3,37 \pm 0,28$ & $2,93 \pm 0,48$ \\
\hline $6 \mathrm{G}^{(2)}$ & $2,09 \pm 0,19$ & $1,88 \pm 0,27$ & $30,00 \pm 2,61$ & $26,25 \pm 3,31$ & $7,78 \pm 0,71$ & $6,65 \pm 0,91$ & $1,27 \pm 0,27 \mathrm{a}$ & $0,57 \pm 0,11 \mathrm{bB}$ & $3,90 \pm 0,64$ & $3,13 \pm 0,52$ \\
\hline $5 \mathrm{G}: 1 \mathrm{~L}$ & $1,95 \pm 0,26$ & $1,90 \pm 0,26$ & $27,17 \pm 2,34$ & $25,50 \pm 3,78$ &, 77 & $6,49 \pm 0,88$ & $1,48 \pm 0,33$ & $1,21 \pm 0,33 \mathrm{~A}$ & $3,57 \pm 0,52$ & $3,13 \pm 0,45$ \\
\hline $4 \mathrm{G}: 2 \mathrm{~L}$ & $2,02 \pm 0,19 \mathrm{a}$ & $1,76 \pm 0,23 b$ & $28,17 \pm 2,14$ & $28,25 \pm 2,40$ & $7,28 \pm 0,66$ & $7,16 \pm 1,15$ & $1,07 \pm 0,21 \mathrm{a}$ & $0,60 \pm 0,14 \mathrm{bB}$ & $3,57 \pm 0,60$ & $3,10 \pm 0,62$ \\
\hline $3 \mathrm{G}: 3 \mathrm{~L}$ & $2,27 \pm 0,27$ & $1,96 \pm 0,23$ & $30,42 \pm 1,96$ & $29,42 \pm 2,42$ & $7,40 \pm 0,88$ & $7,01 \pm 0,96$ & $1,37 \pm 0,46$ & $0,73 \pm 0,25 \mathrm{~B}$ & $3,82 \pm 0,54 \mathrm{a}$ & $3,05 \pm 0,30 \mathrm{~b}$ \\
\hline $2 \mathrm{G}: 4 \mathrm{~L}$ & $1,98 \pm 0,14$ & $1,93 \pm 0,28$ & $27,67 \pm 4,45$ & $28,00 \pm 4,21$ & $6,75 \pm 1,28$ & $7,08 \pm 0,83$ & $0,95 \pm 0,17 \mathrm{a}$ & $0,53 \pm 0,10 \mathrm{bB}$ & $3,74 \pm 1,05$ & $3,02 \pm 0,49$ \\
\hline $1 \mathrm{G}: 5 \mathrm{~L}$ & $2,24 \pm 0,20$ & $1,97 \pm 0,33$ & $32,00 \pm 3,35$ & $27,92 \pm 4,15$ & $7,96 \pm 1,08$ & $7,13 \pm 0,96$ & $1,43 \pm 0,38$ & $1,14 \pm 0,16 \mathrm{~A}$ & $3,50 \pm 0,44 \mathrm{a}$ & $2,84 \pm 0,16 \mathrm{~b}$ \\
\hline $6 \mathrm{~L}^{(3)}$ & $1,95 \pm 0,24$ & $2,08 \pm 0,17$ & $30,83 \pm 2,70$ & $28,90 \pm 2,08$ & $7,19 \pm 1,39 a$ & $6,24 \pm 3,06 \mathrm{~b}$ & $1,15 \pm 0,50 \mathrm{a}$ & $0,58 \pm 0,15 \mathrm{bB}$ & $3,67 \pm 0,46 \mathrm{a}$ & $2,37 \pm 1,18 \mathrm{~b}$ \\
\hline Óleo & ns & ns & ns & ns & ns & ns & ns & $* *$ & ns & ns \\
\hline Período & & \multicolumn{2}{|c|}{$\mathrm{ns}$} & \multicolumn{2}{|c|}{ * } & \multicolumn{2}{|c|}{ * } & \\
\hline $\mathrm{VV}(\%)$ & 10,73 & 12,89 & 10,09 & 11,53 & 13,66 & 12,28 & 27,69 & 20,57 & 16,54 & 14,23 \\
\hline
\end{tabular}

${ }^{(1)}$ Basal, sem adição de óleo; G, óleo de girassol; L, óleo de linhaça. ${ }^{(2)}$ Percentual de óleo de girassol. ${ }^{(3)}$ Percentual de óleo de linhaça. ${ }^{(4)}$ PPT, proteína plasmática total. Médias \pm desvios-padrão seguidas de letras iguais, minúsculas nas linhas e maiúsculas nas colunas, não diferem entre si pelo teste de Tukey,

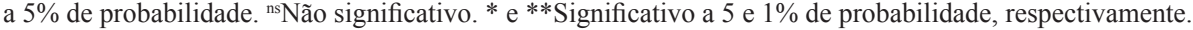


à média para tilápias sadias, conforme Feldman et al. (2006). O valor encontrado neste trabalho é superior aos observados por Falcon et al. (2007), mas similar aos obtidos por Barros et al. $(2002,2004)$ para tilápias hígidas, em condições experimentais semelhantes.

Há ampla variação dos parâmetros hematológicos dos peixes em função de fatores inerentes aos animais ou aos abióticos, como temperatura da água (Ranzini-Paiva \& Silva-Souza, 2004). Foram observadas grandes variações nos parâmetros hematológicos descritos por Ranzini-Paiva \& Silva-Souza (2004) e grande variação na quantificação dos leucócitos dentro do mesmo tratamento, antes e após estímulo pelo frio. Talvez por isso não tenha sido observada redução significativa dos valores médios, apenas dos absolutos, desta variável em todos os tratamentos, ao comparar-se os períodos. As variações inerentes aos peixes são representadas pelo desvio padrão em cada tratamento e o fator abiótico pelos efeitos sobre as variáveis antes e após estímulo pelo frio (Tabela 2).

Ao comparar-se os períodos, os peixes submetidos ao frio alimentados com as dietas $6 \mathrm{G}, 4 \mathrm{G}: 2 \mathrm{~L}, 2 \mathrm{G}: 4 \mathrm{~L}$ e 6L apresentaram menor número de leucócitos. Os peixes submetidos ao frio que consumiram as dietas 6G, 4G:2L, 3G:3L, 2G:4L e 6L também apresentaram menor número de leucócitos, em comparação aos que consumiram a dieta basal. Entre as células da série branca ou leucocitária, os linfócitos são os mais frequentes em sangue periférico de inúmeras espécies de peixe (Ranzini-Paiva \& Silva-Souza, 2004). Por isso, a redução do número de linfócitos seria mais facilmente percebida se houvesse sido feita diferenciação celular neste trabalho. A leucopenia, também determinada de linfopenia, foi demonstrada em estudos com nutrição de peixes por Falcon (2008), Fernandes Junior (2010) e Signor et al. (2010).

Os ácidos graxos poliinsaturados da família ômega-3 têm efeito supressor e os da ômega- 6 efeito modulador do sistema imunológico e, consequentemente, estimulador da proliferação de linfócitos (Curi et al., 2001). Pode-se inferir que a possível linfopenia determinou a redução do total de leucócitos dos peixes que consumiram a dieta com $6 \%$ de óleo de linhaça. Como não houve um padrão de resposta, não há como inferir sobre a redução ocorrida nos peixes que consumiram 6\% de óleo de girassol. Curi et al. (2001) afirmam que a resposta imune requer inicialmente uma resposta inflamatória, com recrutamento de leucócitos, e que a eficiência desta resposta depende de seu reconhecimento e da sua amplificação, com indução rápida e eficiente da proliferação de linfócitos. Mourente et al. (2005) observaram diminuição significativa no número de leucócitos circulantes em robalos (Dicentrarchus labrax L.) alimentados com óleos vegetais (canola, linhaça e oliva), em comparação aos que receberam dieta com óleo de peixe. O menor número de leucócitos poderia reduzir a capacidade de resposta imune dos peixes. Ferreira et al. (2011) também demonstraram que dietas com $5 \%$ de óleo de linhaça são imunossupressoras para tilápias-do-nilo, o que confirma a inferência do presente trabalho.

Feldman et al. (2006) associaram estresse por temperatura com modificação da concentração de leucócitos, sobretudo por afetar a osmorregulação dos peixes e resultar em hemoconcentração ou hemodiluição. Quadros de leucopenia em tilápias-do-nilo, em decorrência de situações de estresse por baixas temperaturas, também foram descritos anteriormente por Atwood et al. (2003), Falcon et al. (2008), Fernandes Junior (2010) e Signor et al. (2010), o que confirma que a temperatura pode exercer efeito determinante sobre os parâmetros sanguíneos dos peixes, mesmo em estado de nutrição adequado.

Atwood et al. (2003) constataram que não há influência da fonte de óleo sobre a aclimatação dos peixes à baixa temperatura, mas que há alterações fisiológicas, como o aumento da glicose e a redução da concentração de sódio sérico. No presente trabalho, peixes alimentados com dietas que continham apenas óleo de linhaça apresentaram menor resistência à redução da temperatura, o que contraria a afirmação desses autores. De acordo com Curi et al. (2001), a secreção de insulina é estimulada primordialmente por substratos energéticos metabolizáveis e pode ser mediada por glicose, o principal agente estimulador, e por ácidos graxos, o que exerce efeito inibitório e cíclico sobre a glicose circulante. Portanto, nutrientes que interfiram de alguma forma no metabolismo da glicose ou de minerais, como o sódio, podem influenciar a aclimatação dos peixes à redução da temperatura.

A concentração de PPT não foi afetada pelo consumo das dietas e variou entre 2,37 e 3,93 $\mathrm{g} \mathrm{dL}^{-1}$, tendo sido reduzida pelo frio nos peixes que consumiram as dietas 3G:3L, 1G:5L e 6L (Tabela 2). Valores médios de referência para tilápias saudáveis foram obtidos por Hrubec et al. (2000) em tilápias híbridas (Oreochromis 
niloticus x híbrido entre $O$. mossambicus x $O$. aureus), por Chen et al. (2003) em tilápias-do-nilo e por Mauel et al. (2007) em tilápias híbridas (O. aureus x O. niloticus), com médias de 3,0 a 7,7 $\mathrm{g} \mathrm{dL}^{-1}$.

O VCM e a CHCM não foram alterados pelos tratamentos, antes ou após estímulo pelo frio, e não foi observado efeito do estímulo pelo frio nestas variáveis (Tabela 3).

Tabela 3. Índices hematimétricos absolutos $(\mathrm{n}=6$; médias \pm desvios-padrão) de tilápias-do-nilo alimentadas com rações com óleos de girassol, linhaça e suas misturas antes e após estímulo pelo frio.

\begin{tabular}{|c|c|c|c|c|}
\hline \multirow[t]{2}{*}{ Tratamento $^{(1)}$} & \multicolumn{2}{|c|}{$\mathrm{VCM}^{(4)}(\mathrm{fL})^{(5)}$} & \multicolumn{2}{|c|}{$\mathrm{CHCM}^{(6)}(\%)$} \\
\hline & Antes & Após & Antes & Após \\
\hline$\overline{\text { Basal }}$ & $155,31 \pm 18,85$ & $151,36 \pm 10,85$ & $36,73 \pm 4,23$ & $38,04 \pm 3,21$ \\
\hline $6 \mathrm{G}^{(2)}$ & $144,05 \pm 10,93$ & $141,58 \pm 23,03$ & $37,33 \pm 2,46$ & $35,56 \pm 3,13$ \\
\hline 5G:1L & $140,32 \pm 9,91$ & $134,16 \pm 7,51$ & $35,28 \pm 1,80$ & $34,28 \pm 2,96$ \\
\hline $4 \mathrm{G}: 2 \mathrm{~L}$ & $139,93 \pm 12,58$ & $163,20 \pm 25,60$ & $36,16 \pm 3,64$ & $40,94 \pm 6,50$ \\
\hline $3 \mathrm{G}: 3 \mathrm{~L}$ & $135,21 \pm 12,15$ & $151,94 \pm 25,08$ & $32,80 \pm 3,62$ & $36,34 \pm 4,50$ \\
\hline $2 \mathrm{G}: 4 \mathrm{~L}$ & $139,68 \pm 18,12$ & $145,59 \pm 10,26$ & $34,05 \pm 5,29$ & $36,98 \pm 2,80$ \\
\hline $1 \mathrm{G}: 5 \mathrm{~L}$ & $142,99 \pm 13,60$ & $143,13 \pm 22,96$ & $35,53 \pm 4,11$ & $36,50 \pm 4,78$ \\
\hline $6 \mathrm{~L}^{(3)}$ & $159,11 \pm 13,11$ & $140,09 \pm 14,87$ & $37,15 \pm 7,41$ & $36,25 \pm 2,64$ \\
\hline Óleo & ns & ns & ns & ns \\
\hline Período & \multicolumn{2}{|c|}{ ns } & \multicolumn{2}{|c|}{ ns } \\
\hline CV (\%) & 9,67 & 12,87 & 8,83 & 10,56 \\
\hline
\end{tabular}

${ }^{(1)}$ Basal, sem adição de óleo; G, óleo de girassol; L, óleo de linhaça. ${ }^{(2)}$ Percentual de óleo de girassol. ${ }^{(3)}$ Percentual de óleo de linhaça. ${ }^{(4)}$ Volume corpuscular médio $(\mathrm{VCM})=[$ (hematócrito $\mathrm{x} 10) /$ eritrócitos $]$. ${ }^{(5)} \mathrm{fL}=10^{-15} \mathrm{~L} .{ }^{(6)}$ Concentração de hemoglobina corpuscular média $(\mathrm{CHCM})=[($ hemoglobina/hematócrito $) \times 100] .{ }^{\text {ns Não significativo. }}$
Os LT, TRG, COLT e C-HDL plasmáticos das tilápias não foram afetados pelo consumo das dietas, independentemente da temperatura. Ao comparar-se as variáveis entre os períodos, os TRG foram reduzidos apenas nos peixes que consumiram a dieta $1 \mathrm{G}: 5 \mathrm{~L}$ e o COLT diminuiu apenas nos peixes que consumiram a dieta $6 \mathrm{~L}$, após estímulo pelo frio (Tabela 4).

Os valores médios de colesterol sanguíneo, com exceção do valor mínimo, antes e após estímulo pelo frio, estão entre os valores indicados como normais para tilápias saudáveis após estímulo pelo frio, o que foi observado nos peixes que consumiram a dieta 6L. A determinação de valores médios de colesterol sanguíneo de tilápias saudáveis tem sido objeto de diversos estudos e variam de 88 a $318 \mathrm{mg} \mathrm{dL}^{-1}$ (Hrubec et al., 2000; Chen et al., 2003; Mauel et al., 2007).

Ferreira et al. (2011) obtiveram valores de colesterol sérico total de peixes alimentados com fontes de ômega-6 (óleos de soja e milho) e ômega-3 (óleo de linhaça) inferiores aos observados no presente trabalho, em peixes não desafiados por temperatura. As diferenças nos teores de óleo utilizados, no tempo de administração das dietas e no tamanho dos peixes quando da coleta de sangue para análises podem ter influenciado o COLT e os TRG séricos.

Nogeral, os peixes que consumiram a dieta basal, com $2,54 \%$ de extrato etéreo e isenta dos óleos de girassol e linhaça, mostraram-se preparados para enfrentar o frio, sem qualquer alteração das variáveis estudadas. Entre tilápias-do-nilo alimentadas com dietas que continham

Tabela 4. Parâmetros sanguíneos $(n=6)$ de tilápias-do-nilo alimentadas com rações com óleos de girassol, linhaça e suas misturas antes e após estímulo pelo frio.

\begin{tabular}{|c|c|c|c|c|c|c|c|c|}
\hline \multirow[t]{2}{*}{ Tratamento $^{(1)}$} & \multicolumn{2}{|c|}{ Lipídeos totais $\left(\mathrm{mg} \mathrm{dL}^{-1}\right)$} & \multicolumn{2}{|c|}{ Triglicerídeos $\left(\mathrm{mg} \mathrm{dL}^{-1}\right)$} & \multicolumn{2}{|c|}{ Colesterol total $\left(\mathrm{mg} \mathrm{dL}^{-1}\right)$} & \multicolumn{2}{|c|}{ Colesterol HDL $\left(\mathrm{mg} \mathrm{dL}^{-1}\right)$} \\
\hline & Antes & Após & Antes & Após & Antes & Após & Antes & Após \\
\hline Basal & $531,13 \pm 168,19$ & $691,10 \pm 205,76$ & $233,67 \pm 124,17$ & $178,50 \pm 65,30$ & $134,50 \pm 29,79$ & $124,00 \pm 32,30$ & $47,67 \pm 12,14$ & $43,17 \pm 10,19$ \\
\hline $6 \mathrm{G}^{(2)}$ & $830,74 \pm 330,16$ & $746,67 \pm 195,79$ & $321,67 \pm 164,42$ & $212,67 \pm 15,91$ & $151,83 \pm 57,58$ & $110,00 \pm 21,36$ & $54,67 \pm 20,25$ & $41,17 \pm 13,93$ \\
\hline $5 \mathrm{G}: 1 \mathrm{~L}$ & $628,25 \pm 143,39$ & $897,78 \pm 244,08$ & $332,00 \pm 188,74$ & $359,83 \pm 245,98$ & $112,00 \pm 28,16$ & $144,33 \pm 59,75$ & $44,17 \pm 13,23$ & $40,00 \pm 16,80$ \\
\hline $4 \mathrm{G}: 2 \mathrm{~L}$ & $682,97 \pm 201,10$ & $800,00 \pm 280,57$ & $287,16 \pm 167,30$ & $208,00 \pm 151,47$ & $137,17 \pm 37,31$ & $118,67 \pm 27,85$ & $40,83 \pm 19,88$ & $50,17 \pm 7,14$ \\
\hline $3 \mathrm{G}: 3 \mathrm{~L}$ & $756,78 \pm 434,82$ & $757,77 \pm 147,14$ & $350,00 \pm 286,73$ & $238,66 \pm 109,36$ & $151,00 \pm 56,20$ & $126,17 \pm 17,79$ & $43,83 \pm 20,14$ & $44,67 \pm 4,23$ \\
\hline $2 \mathrm{G}: 4 \mathrm{~L}$ & $693,87 \pm 407,92$ & $666,67 \pm 111,55$ & $344,17 \pm 292,80$ & $174,67 \pm 45,99$ & $138,17 \pm 42,98$ & $105,67 \pm 27,98$ & $44,67 \pm 11,20$ & $38,50 \pm 9,95$ \\
\hline $1 \mathrm{G}: 5 \mathrm{~L}$ & $687,58 \pm 208,66$ & $662,22 \pm 109,54$ & $290,67 \pm 148,51 \mathrm{a}$ & $160,50 \pm 36,82 b$ & $136,50 \pm 39,32$ & $103,00 \pm 15,13$ & $39,67 \pm 12,37$ & $39,67 \pm 10,27$ \\
\hline $6 \mathrm{~L}^{(3)}$ & $642,88 \pm 203,82$ & $657,77 \pm 100,04$ & $256,20 \pm 73,25$ & $171,67 \pm 32,23$ & $139,40 \pm 30,16 \mathrm{a}$ & $95,00 \pm 18,83 \mathrm{~b}$ & $57,00 \pm 16,80$ & $47,50 \pm 7,45$ \\
\hline Óleo & ns & ns & ns & ns & ns & ns & ns & ns \\
\hline Período & & & \multicolumn{2}{|c|}{$*$} & \multicolumn{2}{|c|}{ ns } \\
\hline CV (\%) & 42,97 & 25,43 & 65,14 & 59,74 & 30,86 & 26,48 & 35,96 & 24,71 \\
\hline
\end{tabular}

${ }^{(1)}$ Basal, sem adição de óleo; G, óleo de girassol; L, óleo de linhaça. ${ }^{(2)}$ Percentual de óleo de girassol. ${ }^{(3)}$ Percentual de óleo de linhaça. Médias \pm desvios padrão

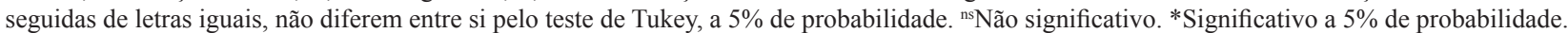


diferentes teores de lipídeos, as que consumiram dieta isenta de suplementação, contendo $2,79 \%$ de extrato etéreo, apresentaram padrão hematológico semelhante (Falcon et al., 2007, 2008). Embora a quantidade de lipídeos nas dietas tenha influência sobre a saúde dos peixes, não há valores estabelecidos na literatura acerca da quantidade adequada para manutenção do estado de higidez de tilápias.

Os peixes que consumiram dietas com $6 \%$ de óleo de linhaça, rica em ácido linolênico e com menor relação entre ácidos graxos poliinsaturados ômega- 6 e ômega-3, apresentaram menor resposta ao estímulo pelo frio. Houve declínio em um maior número de variáveis, como taxa de hemoglobina, número de leucócitos, concentração de proteína plasmática e COLT, ao comparar-se os valores médios anteriores e posteriores ao estímulo pelo frio. Como não foi verificada influência das dietas para peixes não expostos ao frio, não houve influência da relação destes ácidos graxos nos teores avaliados, nos parâmetros hematológicos nem nos lipídeos plasmáticos para a criação em situações de temperatura adequada.

\section{Conclusões}

1. Para tilápias-do-nilo, criadas em condições ótimas de temperatura, as dietas estudadas são adequadas à manutenção do padrão hematológico e dos lipídeos séricos indicados como de peixes saudáveis.

2. Para tilápias-do-nilo submetidas ao frio, a dieta basal, isenta de óleos, é adequada para manutenção da higidez; as dietas com óleos de girassol e linhaça, principalmente as com baixas relações entre ômega-6 e ômega-3, acarretam redução do padrão hematológico.

\section{Agradecimentos}

À Fundação de Amparo à Pesquisa do Estado de Alagoas, pela concessão de bolsa de doutorado ao primeiro autor; ao Conselho Nacional de Desenvolvimento Científico e Tecnológico, pelo auxílio financeiro; à Piscicultura Fernandes, pela doação dos peixes; à Agrocosta - Sementes e Nutrição Animal, pelo fornecimento do óleo de linhaça; ao professor Doutor Carlos Roberto Padovani do Instituto de Biociências da Universidade Estadual Paulista (Unesp), pelo auxílio nas análises estatísticas; e à equipe do Laboratório de Nutrição e Saúde de Peixes da Unesp, pelo auxílio na realização deste trabalho.

\section{Referências}

ATWOOD, H.L.; TOMASSO, J.R.; WEEB, K.; GATLIN III, D.M. Low-temperature tolerance of Nile tilapia, Oreochromis niloticus: effects of environmental and dietary factors. Aquaculture Research, v.34, p.241-251, 2003.

BARROS, M.M.; PEZZATO, L.E.; HISANO, H.; FALCON, D.R.; SÁ, M.V. do C. Farinha de sangue tostada e dietas práticas para tilápia do Nilo (Oreochromis niloticus L.). Acta Scientiarum. Animal Sciences, v.26, p.5-13, 2004.

BARROS, M.M.; PEZZATO, L.E.; KLEEMANN, K.; HISANO, H.; ROSA, G.J.M. Níveis de Vitamina C e Ferro para Tilápia do Nilo (Oreochromis niloticus). Revista Brasileira de Zootecnia, v.31, p.2149-2156, 2002.

BARROS, M.M.; RANZANI-PAIVA, M.J.T.; PEZZATO, L.E.; FALCON, D.R.; GUIMARÃES, I.G. Haematological response and growth performance of Nile tilapia (Oreochromis niloticus L.) fed diets containing folic acid. Aquaculture Research, v.40, p.895-903, 2009.

BELL, M.V.; HENDERSON, R.J.; SARGENT, J.R. The role of polyinsaturated fatty acids in fish. Comparative Biochemistry and Physiology Part B: Comparative Biochemistry, v.83, p.711-719, 1986.

BOYD, C.E.; TUCKER, C.S. Pond aquaculture quality management. Massachusetts: Norwell, 1998. 685p.

BRETT, M.; MÜLLER- NAVARRA, D. The role of highly unsaturated fatty acids in aquatic foodweb processes. Freshwater Biology, v.38, p.483-499, 1997.

CHEN, C.Y.; WOOSTER, G.A.; GETCHELL, R.G.; BOWSER, P.R.; TIMMONS, M.B. Blood chemistry of healthy, nephrocalcinosis-affected and ozone-treated tilapia in a recirculation system, with application of discriminant analysis. Aquaculture, v.218, p.89-102, 2003.

COLLIER, H.B. Standardization of blood haemoglobin determinations. Canadian Medical Association Journal, v.50, p.550-552, 1944.

CURI, R.; POMPÉIA, C.; MIYASAKA, C.K.; ARAÚJO FILHO, J.P. Entendendo a gordura: os ácidos graxos. São Paulo: Manole, 2001. 580p.

FALCON, D.R.; BARROS, M.M.; PEZZATO, L.E.; SOLARTE, W.V.N.; GUIMARÃES, I.G. Leucograma da tilápia-do-Nilo arraçoada com dietas suplementadas com níveis de vitamina $\mathrm{C}$ e lipídeo submetidas a estresse por baixa temperatura. Ciência Animal Brasileira, v.9, p.543-551, 2008.

FALCON, D.R.; BARROS, M.M.; PEZZATO, L.E.; VALLE, J. de $\mathrm{B}$. Lipídeo e vitamina $\mathrm{C}$ em dietas preparatórias de inverno para tilápias-do-nilo. Revista Brasileira de Zootecnia, v.36, p.1462-1472, 2007.

FELDMAN, B.F.; ZINKL, J.G.; JAIN, C.N. Schalm's veterinary hematology. $5^{\text {th }}$ ed. Philadelphia: Lippincott Willians and Wilkins, 2006. 1344p.

FERNANDES JUNIOR, A.C.; PEZZATO, L.E.; GUIMARÃES, I.G.; TEIXEIRA, C.P.; KOCH, J.F.A.; BARROS, M.M. Resposta hemática de tilápias-do-nilo alimentadas com dietas suplementadas 
com colina e submetidas a estímulo por baixa temperatura. Revista Brasileira de Zootecnia, v.39, p.1619-1625, 2010.

FERREIRA, M.W.; ARAÚJO, F.G. de; COSTA, D.V.; LOGATO, P.R.V.; FIGUEIREDO, H.C.P.; MURGAS, L.D.S. Influence of dietary oil sources on muscle composition and plasma lipoprotein concentrations in Nile Tilapia, Oreochromis niloticus. Journal of the World Aquaculture Society, v.42, p.24-33, 2011.

FRINGS, C.S.; FENDLEY, T.W.; DUNN, R.T.; QUEEN, C.A. Improved determination of total serum lipids by the sulfo-phosph-vanilin reaction. Clinical Chemistry, v.18, p.673, 1972.

GOLDENFARB, P.B.; BOWYER, F.P.; HALL, E.; BROSIUS, E. Reproducibility in the hematology laboratory: the microhematocrit determination. American Journal of Clinical Pathology, v.56, p.35-39, 1971.

HRUBEC, T.C.; CARDINALE, J.L.; SMITH, S.A. Hematology and plasma chemistry reference intervals for cultured tilapia (Oreochromis hybrid). Veterinary Clinical Pathology, v.29, p.7-12, 2000.

HRUBEC, T.C.; SMITH, S.A. Hematology of fish. In: FELDMAN, B.F.; ZINKL, J.G.; JAIN, C.N. Schalm's veterinary hematology. $5^{\text {th }}$ ed. Philadelphia: Lippincott Willians and Wilkins, 2006. p.1120-1125.

JAIN, N.C. Schalm's veterinary hematology. $4^{\text {th }}$ ed. Philadelphia: Lea and Febiger, 1986. 1221p.

JOHNSON, R.A.; WICHERN, D.W. Applied multivariate statistical analyses. $5^{\text {th }}$ ed. New Jersey: Prentice-Hall, 2002. $767 \mathrm{p}$.

LIM, C.; WEBSTER, C.D. (Ed.). Tilapia: biology, culture, and nutrition. New York: Food Products Press, 2006. 678p.
MAUEL, M.J.; MILLER, D.L.; MERRILL, A.L. Hematologic and plasma biochemical values of health hybrid tilapia (Oreochromis aureus $\mathrm{x}$ Oreochromis nilotica) maintained in a recirculating system. Journal of Zoo and Wildlife Medicine, v.38, p.420-424, 2007.

MONTERO, D.; KALINOWSKI, T.; OBACH, A.; ROBAINA, L.; TORT, L.; CABALLERO, M.J.; IZQUIERDO, M.S. Vegetable lipid sources for gilthead seabream (Sparus aurata): effects on fish health. Aquaculture, v.225, p.353-370, 2003.

MOURENT, G.; GOOD, J.E.; BELL, J.G. Partial substitution of fish oil with rapessed, linseed and olive oils in diets for European sea bass (Dicentrarchus labrax L.): effects on flesh fatty acid composition, plasma prostaglandins $\mathrm{E}_{2}$ and $\mathrm{F}_{2 \alpha}$, immune function and effectiveness of a fish oil finishing diet. Aquaculture Nutrition, V.11, p.25-40, 2005.

RANZINI-PAIVA, M.J.T.; SILVA-SOUZA, A.T. Hematologia de peixes brasileiros. In: RANZINI-PAIVA, M.J.T.; TAKEMOTO, R.M.; LIZAMA, M. Sanidade de organismos aquáticos. São Paulo: Varela, 2004. p.89-120.

SIGNOR, A.; PEZZATO, L.E.; FALCON, D.R.; GUIMARÃES, I.G.; BARROS, M.M. Parâmetros hematológicos da tilápia-do-Nilo: efeito da dieta suplementada com levedura e zinco e do estímulo pelo frio. Ciência Animal Brasileira, v.11, p.509-519, 2010.

WINTROBE, M.M. Variations in the size and hemoglobin content of erythrocytes in the blood of various vertebrates. Folia Haematologica, v.51, p.32-49, 1934.

YILDIRIM-AKSOY, M.; LIM, C.; DAVIS, D.A.; SHELBY, R.; KLESIUS, P.H. Influence of dietary lipid sources on the growth performance, immune response and resistance of Nile tilapia, Oreochromis niloticus, to Streptococcus iniae Challenge. Journal of Applied Aquaculture, v.19, p.29-49, 2007.

Recebido em 22 de setembro de 2010 e aprovado em 7 de fevereiro de 2011 